\title{
Right Coronary Artery May Grow Even Better than Normal After Arterial Switch Operation for Complete Transposition of the Great Arteries
}

\section{Daisuke Machida}

Yokohama City University Hospital: Yokohama Shiritsu Daigaku Fuzoku Byoin https://orcid.org/00000002-2277-3042

\section{Yukihisa Isomatsu}

Yokohama City University Hospital: Yokohama Shiritsu Daigaku Fuzoku Byoin

\section{Motohiko Goda ( $\sim$ go.supra1203@gmail.com )}

Yokohama City University Hospital: Yokohama Shiritsu Daigaku Fuzoku Byoin

\section{Shinichi Suzuki}

Yokohama City University: Yokohama Shiritsu Daigaku

\section{Keiichiro Kasama}

Yokohama City University: Yokohama Shiritsu Daigaku

\section{Tomoyuki Minami}

Yokohama City University: Yokohama Shiritsu Daigaku

\section{Shota Yasuda}

Yokohama City University Medical Center: Yokohama Shiritsu Daigaku Fuzoku Shimin Sogo Iryo Center

\section{Tomoki Choh}

Yokohama City University Medical Center: Yokohama Shiritsu Daigaku Fuzoku Shimin Sogo Iryo Center

\section{Masami Goda}

Yokohama City University School of Medicine Graduate School of Medicine: Yokohama Shiritsu

Daigaku Igakubu Daigakuin Igaku Kenkyuka

\section{Norihisa Tominaga}

Yokohama City University Hospital: Yokohama Shiritsu Daigaku Fuzoku Byoin

\section{Shotaro Kaneko}

Yokohama City University Hospital: Yokohama Shiritsu Daigaku Fuzoku Byoin

\section{Munetaka Masuda}

Yokohama City University Hospital: Yokohama Shiritsu Daigaku Fuzoku Byoin

\section{Research article}

Keywords: arterial switch operation, complete transposition of the great arteries, Jatene operation, coronary artery 
Posted Date: October 26th, 2020

DOl: https://doi.org/10.21203/rs.3.rs-96030/v1

License: (c) (i) This work is licensed under a Creative Commons Attribution 4.0 International License. Read Full License 
1 (1) Title page

2 Title; Right coronary artery may grow even better than normal after arterial switch

3 operation for complete transposition of the great arteries

4 Authors; Daisuke Machida ${ }^{1}$, Yukihisa Isomatsu ${ }^{1}$, Motohiko Goda ${ }^{1}$, Shinichi Suzuki ${ }^{1}$,

5 Keiichiro Kasama ${ }^{1}$, Tomoyuki Minami², Shota Yasuda², Tomoki Choh², Masami Goda ${ }^{1}$,

6 Norihisa Tominaga ${ }^{1}$, Shotaro Kaneko ${ }^{1}$, Munetaka Masuda $^{1}$

71 Yokohama City University Hospital, Department of Cardiovascular Surgery

82 Yokohama City University Medical Center, Department of Cardiovascular Surgery

9 D. Machida and Y. Isomatsu equally contributed to this work.

11 Corresponding author. Motohiko Goda,

12 Department of Cardiovascular Surgery, Yokohama City University Hospital

13 Fukuura 3-9, Kanazawa-ku, Yokohama, Kanagawa 236-0008, Japan

14 TEL; +81-45-787-2645 FAX; +81-45-786-0226

15 e-mail; mmasuda@yokohama-cu.ac.jp

16

17 Words count. 2575 words 
18 (2) Abstract

19 Background: It is unclear if coronary arteries properly grow in patients who underwent

20 arterial switch operation for complete transposition of the great arteries. The purpose of

21 this study was to clarify the mode of coronary growth and size in these patients.

22 Methods: Eighteen patients who underwent arterial switch operation for complete

23 transposition of the great arteries from 2000 to 2012 in our institution, and in whom

24 coronary angiography was performed in late operative phase, were enrolled in this study.

25 Growth of coronary arteries was evaluated by cubage of coronary arteries based on

26 analyses with coronary angiography. Coronary arteries were divided into small segments

27 and each segment was approximated by a truncated right circular cone. The sum of the

28 cubage of each truncated cone in one coronary artery was approximated as total cubage

29 of the coronary. the coronary cubage index was then calculated by dividing total cubage

30 of a coronary artery by the patient's body surface area. The coronary cubage indexes of

31 the enrolled patients were compared with that of control patients with healed Kawasaki

32 disease.

33 Results: The left coronary cubage indexes of the complete transposition of the great

34 arteries group and the control group were $1.05 \pm 0.34$ and $0.94 \pm 0.34(p=0.598)$, 
35 respectively, and no significant deference was found between groups. On the contrary,

36 the right cubage index of the complete transposition of great arteries group was

37 significantly larger than the control group $(1.08 \pm 0.44$ and $0.54 \pm 0.37$, respectively;

$38 \mathrm{p}=0.007$ ), and total coronary cubage index (left coronary index + right coronary index) of

39 the complete transposition of the great arteries group was also larger than the control

40 group as well $(2.13 \pm 0.7$ and $1.47 \pm 0.6$, respectively; $p=0.026)$.

41 Conclusion: The left coronary arteries after arterial switch operation for complete

42 transposition of great arteries grow as large as normal; however, the right coronary

43 arteries possibly grow even larger.

44 311words

45

46 Key words: arterial switch operation; complete transposition of the great arteries; Jatene

47 operation; coronary artery; 
52 (3) Main text

\section{Introduction}

54 Complete transposition of the great arteries ( $d$-TGA) can be safely corrected in neonates

55 by the arterial switch operation (ASO) and long-term survival is satisfyingly good $[1,2]$.

56 On the contrary, concerns regarding coronary arteries after ASO still last; Turner reported

57 that myocardial flow reserve may be impaired in patients with anatomic left anterior

58 descending artery (LAD) abnormalities [3], and Mavroudis reported that one-third of

59 patients who underwent ASO required reoperations for lesions related with coronary

60 arteries [4]. Particularly, the size of left coronary artery has often been studied and

61 discussed $[1,5,6]$ because it potentially might cause failing ventricle, however the clinical

62 question if coronary arteries properly grow in patients who underwent ASO is still unclear.

63 The purpose of this study was to clarify the mode of coronary growth and size in these

64 patients.

65

66 Patients and Methods

67 Study design and patient population

68 This was a retrospective cohort study performed at a single Japanese center in human 
69 subjects. We identified 18 patients who underwent ASO for d-TGA from 2000 to 2012 in

70 our institution, and in whom coronary angiography (CAG) was performed in late

71 operative phase; in 4-7 years after ASO to evaluate coronary growth and pathology.

72 Mean age and body surface area (BSA) aged 11-191 days (median;18 days) at the

73 ASO. Eleven of them had d-TGA with intact ventricular septum (d-TGA/IVS) and seven

74 had d-TGA with ventricular septal defect (d-TGA/VSD). Eight patients with Kawasaki

75 disease, who were confirmed to have no coronary pathological lesions by CAG, were

76 selected and also enrolled in this study as age-, and BSA-adjusted control subjects. This

77 study was reviewed and approved by the institutional review board at Yokohama City

78 University. Informed consent was obtained from all patients' parents or legal guardians.

80 Angiographic analyses

81 Growth of coronary arteries was evaluated by cubage of coronary arteries based on

82 analyses with CAG. Coronary arteries were divided into small segments on CAG and

83 each segment was approximated by a truncated right circular cone. A cubage $\left(\mathrm{mm}^{3} ; \mathrm{C}\right)$

84 of each truncated cone can be calculated with proximal and distal radiuses $(\mathrm{mm} ; \mathrm{r}, \mathrm{R})$

85 and length $(\mathrm{mm} ; \mathrm{L})$ of each segment using the following mathematical formula. 


$$
\mathrm{C}=\frac{\pi}{3} \times L \times\left(r^{2}+r R+R^{2}\right) m m^{3}
$$

87 The sum of the cubage of each truncated cone in one coronary artery could be

88 acceptable approximation of total cubage of the coronary (T). (Fig 1)

$89 \mathrm{~T}=\Sigma\left(C_{1}+C_{2}+C_{3}+\cdots+C_{n}\right)$

90 Left main trunk and right coronary artery were analyzed on frontal view, and left anterior

91 descending artery, first diagonal branch and circumflex system were analyzed on lateral

92 view. Figure 2 shows a sample of CAG analysis (Fig 2). Coronary cubage index was

93 calculated by dividing total cubage of a coronary artery by the patient's BSA. BSA $\left(m^{2}\right)$

94 was calculated by multiplying 0.007184 by $W e i g h t(k g)^{0.425}$ and $\operatorname{Height}(\mathrm{cm})^{0.725}$

95 according to the DuBois and DuBois formula [7].

96 Radiuses and lengths were measured on CAG with OsiriX DICOM viewer version 4.1.2

97 (Pixmeo SARL. Bernex, Switzerland). Data collection and cubage calculations were

98 performed with Excel software (Microsoft Corporation, Remond, WA, USA).

99

100 Statistical analysis

101 All statistical analyses were performed with IBM SPSS statics 20. Continuous variables

102 were expressed as mean \pm standard deviation and were compared using the student's $t$ 
103 test. Categorical variables are expressed as absolute numbers or percentages and were

104 compared using chi-square testing. The level of statistical significance was set at $p<0.05$.

105

106 Results

107 Patients demographics

108 The list of all 18 patients with d-TGA is given in Table 1. Briefly, 10 out of 18 patients

109 were boys, the median age and BSA at the time of coronary angiography was 6.2 years

110 and $0.75 \mathrm{~m}^{2}$, respectively (Table 1). The median age at the time of ASO was 18 days

111 (range; 11-48 days), excluding a case who underwent Aubert-Imai operation as a two-

112 stage surgery at the age of 191 days. d-TGA/IVS was diagnosed in 11 patients and d-

113 TGA/VSD was in 7 patients. Coronary anatomies were classified by Shaher's

114 classification [8]; Shaher type 1 was confirmed in 11 patients, type $2 \mathrm{~A}$ in 3 , and type $5 \mathrm{~A}$

115 in 3. The median age and BSA of control (Kawasaki) group was 6.0 years and $0.77 \mathrm{~m}^{2}$,

116 respectively, that were almost same as those of TGA group. No stenosis nor occlusion

117 of coronary artery was detected by CAG in both groups.

119 Coronary cubage indexes (cubage $\left(m m^{3}\right) / B S A\left(m^{2}\right)$ ) 
120 The left coronary cubage indexes of d-TGA group and control group were $1.05 \pm 0.34$

121 and $0.94 \pm 0.34(p=0.598)$, respectively, and no significant difference was found between

122 groups. On the contrary, the right cubage index of d-TGA group was significantly larger

123 than control group $(1.08 \pm 0.44$ and $0.54 \pm 0.37$, respectively; $p=0.007)$, and total

124 coronary cubage index (left coronary index + right coronary index) of d-TGA group was

125 larger than control group as well $(2.13 \pm 0.7$ and $1.47 \pm 0.6$, respectively; $p=0.026)$. (Table

$126 \quad 2)$

127

128 Discussion

129 ASO is a standard surgical strategy for d-TGA described by Jatene and Lecompte $[9,10]$

130 which is technically demanding especially in translocation of coronary arteries. Previous

131 studies reported that there possibly could be abnormal myocardial perfusion in children

132 after ASO [3]; however, the mode of coronary arteries growth and function after ASO are

133 still unclear. Yatsunami showed the left coronary arteries were smaller than normal after

134 ASO and that the dominant, large right coronary artery does not fully compensate [5].

135 On the contrary, Turner advocated that anatomic coronary growth is normal in almost

$13690 \%$ coronary arteries after ASO [3]. Our results of this present study differ from these 
137 previous reports; the left coronary arteries after ASO grew as large as normal (Kawasaki),

138 and the right coronary arteries grew even larger.

139 From the embryologic point of view, the development of the coronary vasculature starts

140 from the appearance of round blood islands on epicardium, and then these islands

141 preferentially develop in the sulci of the heart surface and form a rudimentary plexus

142 [11,12]. This coronary plexus grows and climbs superiorly, and then develops the

143 coronary network. The coronary stems are formed from the coronary network and

144 attaches to the aortic root. Based on the embryologic and anatomical differences of

145 relationship of great arteries and ventricles between the normal and the d-TGA fetus, the

146 reasons underlying our results should be discussed in taking the logical fetal circulation

147 into account. The oxygen saturation of the blood distributed in the ascending aorta is

$14865 \%$, mixture of the blood from foramen ovale with an oxygen saturation of about $85 \%$

149 and that from pulmonary veins with an oxygen saturation of about $60 \%$ in the normal

150 fetus [13]. On the contrary, the blood ejected by the right ventricle to the ascending aorta

151 have an oxygen saturation of about as low as $45 \%$; mixture of blood from placenta with

152 an oxygen saturation of about $85 \%$ and that from systemic circulation with an oxygen

153 saturation of about $35 \%$, that is similar to that of the pulmonary artery in the normal fetus 
154 [13]. Therefore, the oxygen saturation of the blood distributed in the coronary artery of

155 the d-TGA fetus would be lower than that of the normal fetus [13]. Based on this logic,

156 total coronary blood flow of the d-TGA fetus should consequently be 1.5 times higher

157 than that of the normal fetus to provide the proper oxygen delivery. One can then

158 hypotheses that coronary arteries of d-TGA possibly grow the same as or even better

159 than the normal due to the higher coronary flow.

160 Previous studies documented that the right coronary arteries are larger than the left,

161 which consists with our results. It is questioned why this phenomenon happens. The right

162 coronary artery flow has been documented to increase with a positive correlation to

163 systolic right ventricular pressure in children with congenital heart defect [14]. The right

164 ventricles corresponding to systemic circulation in d-TGA should have higher systolic

165 pressure than normal. The right coronary artery flow then consequently increase,

166 therefore, we could hypothesize that it leads to enlarged right coronary arteries.

167 Moreover, the fact that the distance between the right coronary stems and the aortic root

168 which is located anterior to the main pulmonary artery in TGA fetus is shorter than normal

169 might increase the right coronary flow.

170 Hemodynamically and anatomically different circumstances of the coronary artery 
171 development is considered to play major roles for the results of the present study as

172 described above. Although these factors mainly affect within embryos and neonates

173 before correction, we believe that they can substantially impact to the coronary artery

174 growth even years after ASO.

175

176 Study limitations.

177 This study included single center data and the numbers of the subjects are small.

178 Regardless of the type of coronary artery anatomy in d-TGA, the coronary cubage was

179 comprehensively evaluated. But impacts of coronary artery anatomy to the coronary

180 growth could not be evaluated because of small samples. Observational period was as

181 short as 5-7 years in this study. Longer observation of larger cohorts of patients is then

182 necessary to evaluate the coronary artery growth more properly. Also, the impact of

183 embryonic circulation on coronary artery growth even after the corrective surgery has to

184 be studied from the point of gene expression.

186 Conclusion

187 The left coronary arteries after ASO for d-TGA grow as large as normal; however, the 
188 right coronary arteries possibly grow even larger. Further evaluation with long

189 observation of larger cohorts is mandatory for verifying these results.

\section{Abbreviations}

192 d-TGA: Complete transposition of the great arteries, ASO: arterial switch operation, LAD:

193 left descending artery, CAG: coronary angiography, BSA: body surface area, VSD:

194 ventricular septal defect, IVS: intact ventricular septum

195

196 Declarations

197 Ethical Approval and Consent to participate

198 This study was approved by the institutional review board at Yokohama City University.

199 Informed consent was obtained from all patients' parents or legal guardians.

200

201 Consent for publication

202 Consent for publication was obtained from all patients' parents or legal guardians.

203

204 Availability of supporting data 
205 All data are available.

206

207 Competing interests

208 All authors declare that they have no competing interests.

209

210 Funding

211 No funding.

212

213 Authors' contributions

214 DM and YI designed the study. DM and MoG wrote and submitted the manuscript. DM,

$215 \mathrm{YI}, \mathrm{MaG}, \mathrm{NT}, \mathrm{SK}, \mathrm{KK}, \mathrm{TM}, \mathrm{SY}, \mathrm{TC}$ collected and analyzed data together. SS and MM

216 supervised this study. All authors read the final version of this article and approved for

217 publication.

218

219 Acknowledgements

220 Not applicable

221 


\section{Authors' information}

223 Daisuke Machida,

224 Department of Cardiovascular Surgery, Yokohama City University Hospital

225 Fukuura 3-9, Kanazawa-ku, Yokohama, Kanagawa 236-0008, Japan

226 TEL; +81-45-787-2645 FAX; +81-45-786-0226

227 e-mail; dmachida@yokohama-cu.ac.jp

228

229 References

230 [1] Williams WG, McCrindle BW, Ashburn DA, Jonas RA, Mavroudis C, Blackstone EH,

231 the members of the Congenital heart Surgeon's Society. Outcome of 829 neonates with

232 complete transposition of the great arteries 12-17 years after repair. Eur $\mathrm{J}$ Cardiothorac

233 Surg 2003;24:1-10

234 [2] Masuda M, Kado H,Nakajima K, Onizuka T, Kurisu K, Morita S, Shiokawa Y, Imoto

235 Y, Tominaga R, Yasui H. Early and Late Results of Total Correction of Congenital Cardiac

236 Anomalies in Infancy. Jpn J Thorac Cardiovasc Surg. 2001 Aug;49(8):497-503

237 [3] Turner DR, Muzik O, Forbs TJ, Sullivan NM, Singh TP. Coronary diameter and

238 vasodilator function in children following arterial switch operation for complete 
239 transposition of the great arteries. Am j Cardiol 2010;106:421-425

240 [4] Mavroudis C, Stewart RD, Backer CL, Rudra H, Vargo P, Jacobs ML. Reoperative

241 techniques for complications after arterial switch. Ann Thirac Surg 2011;92:1747-1755

242 [5] Yatsunami K, Nakazawa M, Kondo C, Teshima H, Momma K, Takanashi Y, Imai Y.

243 Small left coronary arteries after arterial switch operation for complete transposition. Ann

244 Thorac surg 1997;64:746-751

245 [6] Amin Z, McElhinney DB, Moore P, Reddy VM, Hanley FM. Coronary arterial size late

246 after the atrial intervention procedure for transposition of the great arteries: implications

247 for the arterial switch operation. J Thorac Cardiovasc Surg. 2000;120:1047-1052

248 [7] DuBois D, DuBois EF. A formula to estimate the approximate surface area if height

249 and weight be known. Arch Intern Med 1916;17:863-871

250 [8] Shaher RM, Puddu GC. Coronary arterial anatomy in complete transposition of the

251 great vessels. Am J Cardiol 1966;17:355-361

252 [9] Jatene AD, Fonted VF, Paulista PP, Souza LC, Neger F, Galantier M, Sousa JE.

253 Anatomic correction of transposition of the great vessels. J Thorac Cardiovasc Surg.

$254 \quad 1976 ; 72: 364-370$

255 [10] Lecompte Y, Zannini L, Hanzan E, Jarreau MM, Bex JP, Tu TV, Neveux JY. Anatomic 
256 correction of transposition of the great arteries. J Thorac Cardiovasc Surg 1981;82:629-

257631.

258 [11] Hutching GM, Kessler-Hanna A, Moore GW. Development of the coronary arteries

259 in the embryonic human heart. Circulation 1988;77:1250-1257

260 [12] Goda M, Arakawa K, Yano H, Himeno H, Yamazaki I, Suzuki S, Masuda M.

261 Congenital aortopulmonary fistulas combined with bilateral coronary artery fistulas. Ann

262 Thorac Surg 2011;92:1524-1526

263 [13] Rudolph AM. Aortopulmonary transposition, Congenital Diseases of the Heart. $3^{\text {rd }}$

264 ed. San Francisco: Wiley-Blackwell, 2009. p.465-505

265 [14] Watanabe N, Awa S, Akagi M, Ando Y, Oki N, Watagai T, et al. Effect of heart rate

266 and right ventricular pressure on right coronary arterial flow and its systolic versus

267 diastolic distribution in a variety of congenital heart diseases in children. Pediatr Int.

$268 \quad 2000 ; 42: 476-482$. 


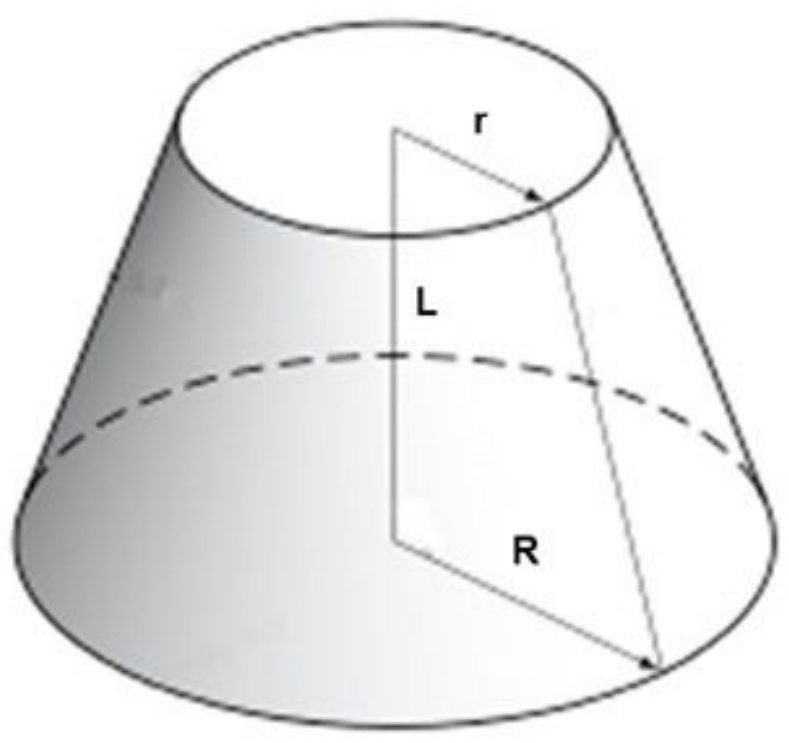

a

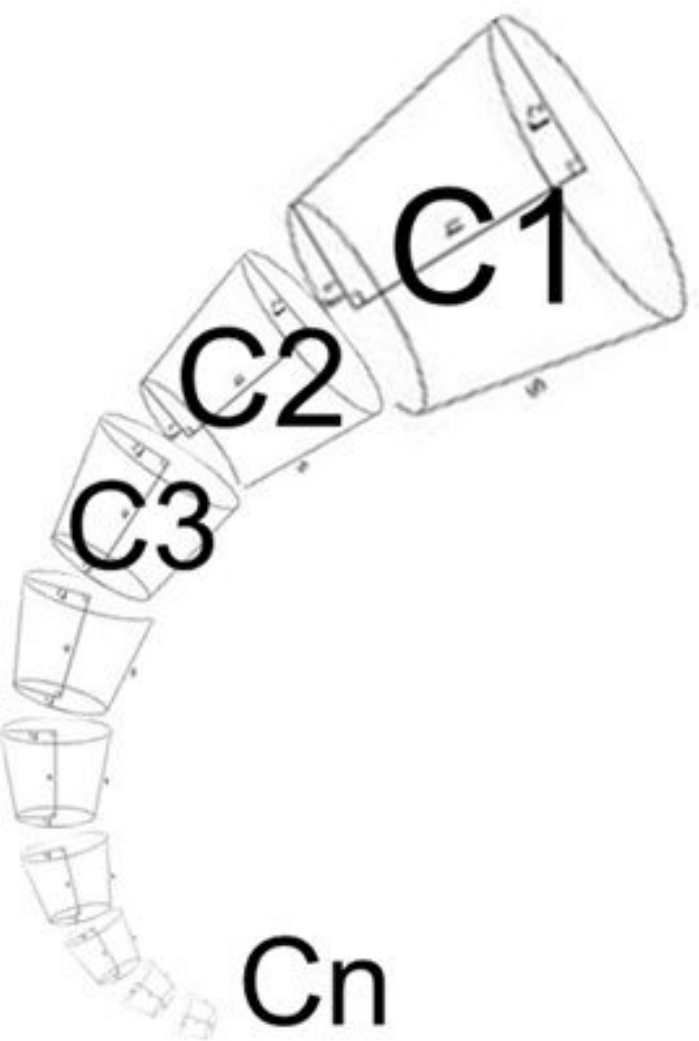

b

\section{Figure 1}

Approximation of coronary arteries by multiple circular truncated cones a. A cubage (C) of each truncated cone can be calculated with proximal and distal radiuses $(r, R)$ and length $(L)$ of each segment using the following mathematical formula. $C=\pi / 3 \times L \times\left(r^{\wedge} 2+r R+R^{\wedge} 2\right) m m^{\wedge} 3 b$. The sum of the cubage of each truncated cone in one coronary artery could be acceptable approximation of total cubage of the coronary $(T) . T=\sum\left(C_{-} 1+C \_-2+C \_3+\square+C \_n\right)$ 


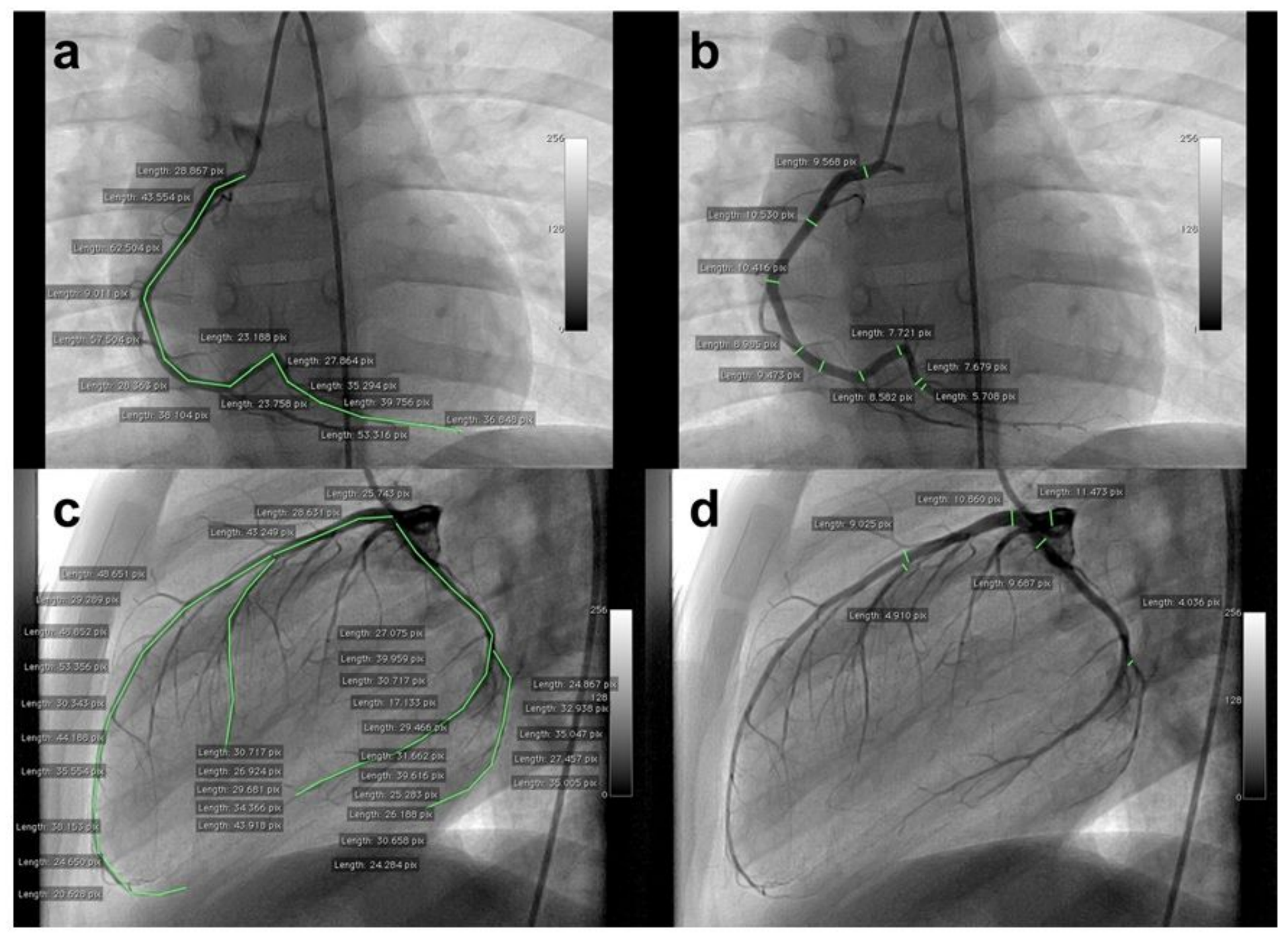

\section{Figure 2}

Angiographical analysis Radiuses and lengths of each segments of coronary arteries were measured on CAG with OsiriX DICOM viewer version 4.1.2. a, b; Right coronary artery and left main trunk were analyzed on frontal view. c, d; Left anterior descending artery, first diagonal branch and circumflex system were analyzed on lateral view.

\section{Supplementary Files}

This is a list of supplementary files associated with this preprint. Click to download.

- Tables.docx 